\title{
Parameters and design optimization of the ring piezoelectric ceramic transformer
}

\author{
Jiří Erhart \\ Measurement Department, VÚTS a.s., Svárovská 619 \\ 46001 Liberec XI, Czech Republic \\ jiri.erhart@tul.cz
}

Received 2 April 2015; Revised 24 June 2015; Accepted 29 June 2015; Published 12 August 2015

\begin{abstract}
Main aim of the presented paper is the theoretical analysis and experimental verification of the transformation parameters for the new type of nonhomogeneously poled ring transformer. The input part is poled in the thickness direction and output part in the radial direction. Two transformer geometries are studied - the input part is at inner ring segment, or it is at the outer ring segment. The optimum electrode size aspect ratios have been found experimentally as $d_{1} / D \approx 0.60-0.65$ for the ring with aspect ratio $d / D=0.2$. The fundamental as well as higher overtone resonances were studied for the transformation ratio, the optimum resistive load, efficiency and no-load transformation ratio. Higher overtones have better transformation parameters compared to the fundamental resonance. The new type ring transformer exhibits very high transformation ratios up to 200 under no-load and up to 13.4 under a high efficiency of $97 \%$ at the optimum load conditions of $10 \mathrm{k} \Omega$. Strong electric field gradient at the output circuit is applicable for the electrical discharge generation.
\end{abstract}

Keywords: Piezoelectric transformer; ring resonator; PZT ceramics.

\section{Introduction}

Piezoelectric transformer (PT) is a resonant device, which uses converse piezoelectric effect in primary circuit (actuator part) and direct piezoelectric effect in secondary circuit (sensor part) for the transformation of $\mathrm{AC}$ electrical signals - see review on modeling of PTs. ${ }^{1}$

One of the most successful transformer designs is called Rosen-type transformer. ${ }^{2}$ It has several modifications in the shape of ceramics plate, e.g., rectangular plate (with thicknesspoled primary circuit and longitudinally poled secondary circuit part) or circular plate (disc plate with thickness-poled primary circuit and radially poled secondary circuit part).

Disc geometry of PTs was studied previously for the "ring-dot" PT, i.e., for the homogeneously (thickness) poled ceramic disc with the electrode divided into the input "dot" and output concentric "ring". ${ }^{3}$ This type of PT exhibits very good transformation ratio, efficiency and simple vibration mode spectrum. The "ring-dot" geometry was also studied for thin PZT ring. ${ }^{4}$ Such PT shows very good performance for the third overtone mode. Homogeneously thickness-poled ring PT with the electrode divided into two parts was studied by the equivalent circuit model. ${ }^{5}$ The transformation ratio, input and output impedance, efficiency, resonance frequency and modal shapes are theoretically derived and verified on the transformer samples.

Ceramic tube geometry was theoretically modeled for the thickness shear mode, ${ }^{6}$ i.e., axially poled two PZT tubes in contact with cylindrical electrodes deposited on the inner and outer circumference faces with the common ground electrode between tubes. However, theoretical results were not verified by any experiment. Similar modeling was performed also for the cylindrical transformer made from two concentric ceramics tubes with radial polarizations and the same electrode design. $^{7}$ Both tubular geometries exhibit only moderate transformation ratio (less than 8 under mechanical losses $Q_{m}=100$ ). Cylindrical geometry of piezoelectric resonator was studied previously for the use of energy harvesting transducer, which converts elastic vibrations propagated through the elastic wall into the electrical signal. ${ }^{8}$ The radially poled piezoelectric tube transformer sandwiched between two metallic tubes was studied for the equivalent circuit. ${ }^{9}$ Similarly, the composite transducer made from the radially poled PZT tube and metallic tube in contact was studied. ${ }^{10}$

In this contribution, we would like to present our study on the parameters of the new type of the ring transformer. Set of samples has been prepared from the hard PZT (type APC841) for the ring transformers with the variable electrode dimensions. The transformation ratio and efficiency are measured for the low-power signal and compared with the derived formulae based on the transformer dimensions and material parameters like the electromechanical coupling factor and permittivity. The transformer parameters are measured as a function of frequency and impedance load in the secondary circuit. The optimum impedance for the maximum efficiency and the optimum electrode aspect ratio for the studied ring size have been found.

This is an Open Access article published by World Scientific Publishing Company. It is distributed under the terms of the Creative Commons Attribution 3.0 (CC-BY) License. Further distribution of this work is permitted, provided the original work is properly cited. 


\section{Theory}

Two different geometries of the ring transformer were studied analytically (see Fig. 1). The ring-type transformer could be designed with the primary part at the center ring [Fig. 1(a) secondary part at the outer ring] or with the primary part at the outer ring [Fig. 1(b) - secondary part at the center ring]. Both designs exhibit piezoelectric ceramics in the primary (input) circuit poled in the thickness direction and the secondary (output) circuit poled in the radial direction. Equations of state for the input part could be expressed in cylindrical coordinates as

$$
\begin{aligned}
& T_{r r}^{(1)}=c_{11}^{P} u_{r, r}^{(1)}+c_{12}^{P} \frac{u_{r}^{(1)}}{r}-e_{31}^{P} E_{3}^{(1)}, \\
& T_{\vartheta \vartheta}^{(1)}=c_{12}^{P} u_{r, r}^{(1)}+c_{11}^{P} \frac{u_{r}^{(1)}}{r}-e_{31}^{P} E_{3}^{(1)}, \\
& D_{3}^{(1)}=e_{31}^{P}\left(u_{r, r}^{(1)}+\frac{u_{r}^{(1)}}{r}\right)+\varepsilon_{33}^{P} E_{3}^{(1)},
\end{aligned}
$$

and for the output part as

$$
T_{r r}^{(2)}=c_{33}^{P} u_{r, r}^{(2)}+c_{13}^{P} \frac{u_{r}^{(2)}}{r}-e_{33}^{P} E_{r}^{(2)},
$$

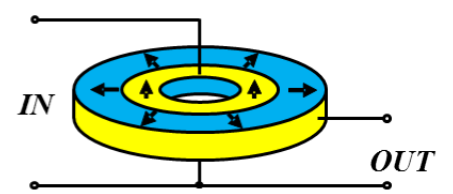

ceramics

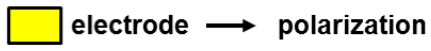

(a)

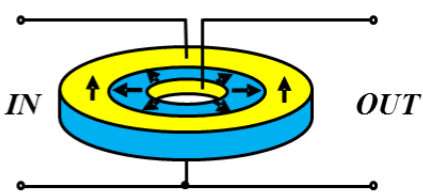

ceramics $\square$ electrode $\longrightarrow$ polarization

(b)

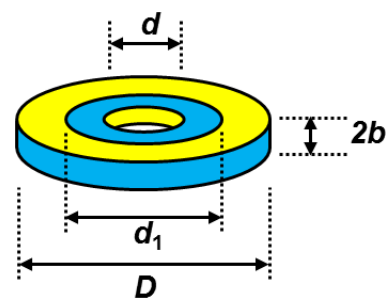

(c)

Fig. 1. Ring-type transformer geometry - (a) inner ring as PT's input, (b) outer ring as PT's input and (c) dimensions.

$$
\begin{aligned}
& T_{\vartheta \vartheta}^{(2)}=c_{13}^{P} u_{r, r}^{(2)}+c_{11}^{P \prime} \frac{u_{r}^{(2)}}{r}-e_{31}^{P \prime} E_{r}^{(2)}, \\
& D_{r}^{(2)}=e_{33}^{P} u_{r, r}^{(2)}+e_{31}^{P \prime} \frac{u_{r}^{(2)}}{r}+\varepsilon_{33}^{P \prime} E_{r}^{(2)},
\end{aligned}
$$

where the planar electromechanical tensor components are defined by

$$
\begin{gathered}
c_{11}^{P}=c_{11}^{E}-\frac{\left(c_{13}^{E}\right)^{2}}{c_{33}^{E}}, \quad c_{11}^{P \prime}=c_{11}^{E}-\frac{\left(c_{12}^{E}\right)^{2}}{c_{11}^{E}}, \\
c_{12}^{P}=c_{12}^{E}-\frac{\left(c_{13}^{E}\right)^{2}}{c_{33}^{E}}, \quad c_{13}^{P}=c_{13}^{E}\left(1-\sigma^{P}\right), \\
c_{33}^{P}=c_{33}^{E}-\frac{\left(c_{13}^{E}\right)^{2}}{c_{11}^{E}}, \quad \sigma^{P}=\frac{c_{12}^{P}}{c_{11}^{P}}, \\
e_{31}^{P}=e_{31}-e_{33} \frac{c_{13}^{E}}{c_{33}^{E}}, \quad e_{31}^{P \prime}=e_{31}\left(1-\sigma^{P}\right), \\
e_{33}^{P}=e_{33}-e_{31} \frac{c_{13}^{E}}{c_{11}^{E}}, \\
\varepsilon_{33}^{P}=\varepsilon_{33}^{S}+\frac{e_{33}^{2}}{c_{33}^{E}}, \quad \varepsilon_{33}^{P \prime}=\varepsilon_{33}^{S}+\frac{e_{31}^{2}}{c_{11}^{E}}
\end{gathered}
$$

and $T, D, u$ are components of the mechanical stress, electrical displacement and mechanical displacement, respectively. Superscripts (1) and (2) denote the primary and secondary parts of the transformer body. The origin of the coordinate system is in the ring center.

The boundary conditions include mechanically free inner and outer ring's circumference and the continuity of the radial components of the mechanical stress and displacement

$$
\begin{gathered}
T_{r r}^{(1)}=0, \quad r=r_{1}=d / 2, \\
T_{r r}^{(1)}=T_{r r}^{(2)}, \quad u_{r}^{(1)}=u_{r}^{(2)}, \quad r=r_{2}=d_{1} / 2, \\
T_{r r}^{(2)}=0, \quad r=r_{3}=D / 2, \\
U_{2} e^{j \omega t}=\Phi^{(2)}\left(r_{3}\right)-\Phi^{(2)}\left(r_{2}\right), \\
E_{3}^{(1)}=-\frac{U_{1}}{2 b} e^{j \omega t}, \quad E_{r}^{(2)}=-\Phi_{, r}^{(2)},
\end{gathered}
$$

for the ring PT design in Fig. 1(a) and

$$
\begin{gathered}
T_{r r}^{(2)}=0, \quad r=r_{1}=d / 2, \\
T_{r r}^{(1)}=T_{r r}^{(2)}, \quad u_{r}^{(1)}=u_{r}^{(2)}, \quad r=r_{2}=d_{1} / 2, \\
T_{r r}^{(1)}=0, \quad r=r_{3}=D / 2, \\
U_{2} e^{j \omega t}=\Phi^{(2)}\left(r_{1}\right)-\Phi^{(2)}\left(r_{2}\right), \\
E_{3}^{(1)}=-\frac{U_{1}}{2 b} e^{j \omega t}, \quad E_{r}^{(2)}=-\Phi_{r}^{(2)},
\end{gathered}
$$

for the ring PT design in Fig. 1(b). An electric field is defined as a derivative of electric potential $\phi$. Radial vibration mode is characterized by the radial displacement component $u_{r}$. Mechanical stresses include only radial $T_{r r}$ and tangential $T_{\vartheta \vartheta}$ components. 
The equation of motion for the radial vibration mode in the cylindrical coordinates

$$
T_{r r, r}^{(i)}+\frac{T_{r r}^{(i)}-T_{\vartheta \vartheta}^{(i)}}{r}=\rho \ddot{u}_{r}^{(i)}, \quad i=1,2
$$

is solved together with Maxwell's equations

$$
D_{3,3}^{(1)}=0, \quad D_{r, r}^{(2)}+\frac{D_{r}^{(2)}}{r}=0
$$

and equations of state (1)-(6). Combination of these equations results in Bessel's equation for the primary circuit

$$
\begin{gathered}
u_{r, \eta \eta}^{(1)}+\frac{u_{r, \eta}^{(1)}}{\eta}+\left(1-\frac{1}{\eta^{2}}\right) u_{r}^{(1)}=0 \\
\eta=2 \pi f r \sqrt{\frac{\rho}{c_{11}^{P}}}
\end{gathered}
$$

and in Lommel's equation for the secondary circuit

$$
\begin{gathered}
u_{r, \eta^{\prime} \eta^{\prime}}^{(2)}+\frac{u_{r, \eta^{\prime}}^{(2)}}{\eta^{\prime}}+\left(1-\frac{\nu^{2}}{\eta^{\prime 2}}\right) u_{r}^{(2)}=\frac{K C^{(2)}}{\eta^{\prime 2}} \\
\eta^{\prime}=2 \pi f r \sqrt{\frac{\rho}{c_{33}^{P}+\frac{\left(e_{33}^{P}\right)^{2 \prime}}{\varepsilon_{33}^{P \prime}}}} \\
\nu^{2}=\frac{c_{11}^{P \prime}+\frac{\left(e_{31}^{P \prime}\right)^{2}}{\varepsilon_{33}^{P \prime}}}{c_{33}^{P}+\frac{\left(e_{33}^{P}\right)^{2}}{\varepsilon_{33}^{P \prime}}}, \quad K=-\frac{e_{31}^{P \prime}}{\varepsilon_{33}^{P \prime}\left(c_{33}^{P}+\frac{\left(e_{33}^{P}\right)^{2}}{\varepsilon_{33}^{P \prime}}\right)} .
\end{gathered}
$$

Solution of Eqs. (17) and (19) could be expressed using combination of Bessel's and Lommel's functions

$$
\begin{gathered}
u_{r}^{(1)}(\eta, t)=\left[A^{(1)} J_{1}(\eta)+B^{(1)} Y_{1}(\eta)\right] e^{j \omega t} \\
u_{r}^{(2)}\left(\eta^{\prime}, t\right)=\left[A^{(2)} J_{\nu}\left(\eta^{\prime}\right)+B^{(2)} Y_{\nu}\left(\eta^{\prime}\right)\right. \\
\left.-K C^{(2)} s_{-1, \nu}\left(\eta^{\prime}\right)\right] e^{j \omega t} \\
s_{-1, \nu}(\eta)=\frac{\pi}{2}\left[J_{\nu}(\eta) \int_{0}^{\eta} \frac{Y_{\nu}(x)}{x} d x-Y_{\nu}(\eta) \int_{0}^{\eta} \frac{J_{\nu}(x)}{x} d x\right] .
\end{gathered}
$$

The integration constants $A^{(1)}, A^{(2)}, B^{(1)}, B^{(2)}$ and $C^{(2)}$ must be calculated from the boundary conditions (10)-(14). The electrical parameters of PT could be further calculated from the currents in the input and output circuits. The input current $I_{1}$ and the output current $I_{2}$ are calculated as the displacement currents

$$
\begin{gathered}
I_{1}=\frac{\partial}{\partial t} \int_{r_{1}}^{r_{2}} D_{3}^{(1)}(b, t) 2 \pi r d r \\
I_{2}=\frac{\partial}{\partial t} \int_{-b}^{+b} D_{r}^{(2)}\left(r_{3}, t\right) 2 \pi r_{3} d x_{3}
\end{gathered}
$$

for the design in Fig. 1(a) and

$$
\begin{gathered}
I_{1}=\frac{\partial}{\partial t} \int_{r_{2}}^{r_{3}} D_{3}^{(1)}(b, t) 2 \pi r d r \\
I_{2}=\frac{\partial}{\partial t} \int_{-b}^{+b} D_{r}^{(2)}\left(r_{1}, t\right) 2 \pi r_{1} d x_{3}
\end{gathered}
$$

for the design in Fig. 1(b). We can express ${ }^{3}$

$$
\begin{aligned}
& U_{2}=\Gamma_{1} U_{1}-Z_{2} I_{2}, \\
& I_{1}=-\frac{U_{1}}{Z_{1}}+\Gamma_{2} I_{2},
\end{aligned}
$$

where $Z_{1}, Z_{2}, \Gamma_{1}$ and $\Gamma_{2}$ are the complex functions of frequency, PT dimensions, electromechanical properties of ceramics and its density $\rho$.

The output current is simply related to the impedance load $Z_{L}$ in the output circuit as

$$
I_{2}=\frac{U_{2}}{Z_{L}}
$$

The transformation ratio could be expressed from the combination of Eqs. (26) and (28) by

$$
\frac{U_{2}}{U_{1}}=\frac{\Gamma_{1} Z_{L}}{Z_{2}+Z_{L}}
$$

Transformation parameters are

$$
\Gamma_{1}=\frac{\alpha_{1}\left(\eta_{1}, \eta_{2}, \eta_{2}^{\prime}, \eta_{3}^{\prime}\right) M_{1}\left(\eta_{3}^{\prime}\right)+\alpha_{3}\left(\eta_{1}, \eta_{2}, \eta_{2}^{\prime}, \eta_{3}^{\prime}\right) M_{2}\left(\eta_{3}^{\prime}\right)}{\alpha_{2}\left(\eta_{1}, \eta_{2}, \eta_{2}^{\prime}, \eta_{3}^{\prime}\right) M_{1}\left(\eta_{3}^{\prime}\right)+\alpha_{4}\left(\eta_{1}, \eta_{2}, \eta_{2}^{\prime}, \eta_{3}^{\prime}\right) M_{2}\left(\eta_{3}^{\prime}\right)}
$$

$$
\begin{aligned}
\frac{1}{Z_{2}}= & -j \omega(4 \pi b) \\
& \times \frac{\alpha_{2}\left(\eta_{1}, \eta_{2}, \eta_{2}^{\prime}, \eta_{3}^{\prime}\right) M_{1}\left(\eta_{3}^{\prime}\right)+\alpha_{4}\left(\eta_{1}, \eta_{2}, \eta_{2}^{\prime}, \eta_{3}^{\prime}\right) M_{2}\left(\eta_{3}^{\prime}\right)}{N\left(\eta_{3}^{\prime}\right)}
\end{aligned}
$$




$$
\begin{aligned}
& \alpha_{1}\left(\eta_{1}, \eta_{2}, \eta_{2}^{\prime}, \eta_{3}^{\prime}\right)=-\frac{e_{31}^{P}}{2 b} \frac{N\left(\eta_{3}^{\prime}\right)}{R\left(\eta_{1}, \eta_{2}, \eta_{2}^{\prime}, \eta_{3}^{\prime}\right)}\left[Y_{1}\left(\eta_{2}\right)\left(L_{1}\left(\eta_{2}\right)-L_{1}\left(\eta_{1}\right)\right)+J_{1}\left(\eta_{2}\right)\left(L_{2}\left(\eta_{1}\right)-L_{2}\left(\eta_{2}\right)\right)\right] \\
& \alpha_{2}\left(\eta_{1}, \eta_{2}, \eta_{2}^{\prime}, \eta_{3}^{\prime}\right)=-\frac{N\left(\eta_{3}^{\prime}\right)}{R\left(\eta_{1}, \eta_{2}, \eta_{2}^{\prime}, \eta_{3}^{\prime}\right)\left[\left(P_{2}\left(\eta_{3}^{\prime}\right)-P_{2}\left(\eta_{2}^{\prime}\right)\right) N\left(\eta_{3}^{\prime}\right)-\left(Q\left(\eta_{3}^{\prime}\right)-Q\left(\eta_{2}^{\prime}\right)\right) M_{2}\left(\eta_{3}^{\prime}\right)\right]} \\
& \times\left[c_{11}^{P} k\left(L_{1}\left(\eta_{2}\right) L_{2}\left(\eta_{1}\right)-L_{1}\left(\eta_{1}\right) L_{2}\left(\eta_{2}\right)\right) \times\left(K s_{-1, \nu}\left(\eta_{2}^{\prime}\right) M_{1}\left(\eta_{3}^{\prime}\right)-Y_{\nu}\left(\eta_{2}^{\prime}\right) N\left(\eta_{3}^{\prime}\right)\right)\right. \\
& \left.-c_{33} k^{\prime}\left(J_{1}\left(\eta_{2}\right) L_{2}\left(\eta_{1}\right)-Y_{1}\left(\eta_{2}\right) L_{1}\left(\eta_{1}\right)\right) \times\left(M_{2}\left(\eta_{3}^{\prime}\right) N\left(\eta_{2}^{\prime}\right)-N\left(\eta_{3}^{\prime}\right) M_{2}\left(\eta_{2}^{\prime}\right)\right)\right] \\
& R\left(\eta_{1}, \eta_{2}, \eta_{2}^{\prime}, \eta_{3}^{\prime}\right)=\left[c_{11}^{P} k\left(L_{1}\left(\eta_{2}\right) L_{2}\left(\eta_{1}\right)-L_{1}\left(\eta_{1}\right) L_{2}\left(\eta_{2}\right)\right)\left(K s_{-1, \nu}\left(\eta_{2}^{\prime}\right) M_{1}\left(\eta_{3}^{\prime}\right)-J_{\nu}\left(\eta_{2}^{\prime}\right) N\left(\eta_{3}^{\prime}\right)\right)\right. \\
& \left.-c_{33} k^{\prime}\left(J_{1}\left(\eta_{2}\right) L_{2}\left(\eta_{1}\right)-Y_{1}\left(\eta_{2}\right) L_{1}\left(\eta_{1}\right)\right)\left(M_{1}\left(\eta_{3}^{\prime}\right) N\left(\eta_{2}^{\prime}\right)-N\left(\eta_{3}^{\prime}\right) M_{1}\left(\eta_{2}^{\prime}\right)\right)\right] \\
& +\frac{\alpha_{3}\left(\eta_{1}, \eta_{2}, \eta_{2}^{\prime}, \eta_{3}^{\prime}\right)}{\alpha_{1}\left(\eta_{1}, \eta_{2}, \eta_{2}^{\prime}, \eta_{3}^{\prime}\right)}\left[c _ { 1 1 } ^ { P } k ( L _ { 1 } ( \eta _ { 2 } ) L _ { 2 } ( \eta _ { 1 } ) - L _ { 1 } ( \eta _ { 1 } ) L _ { 2 } ( \eta _ { 2 } ) ) \left(K s_{-1, \nu}\left(\eta_{2}^{\prime}\right) M_{2}\left(\eta_{3}^{\prime}\right)\right.\right. \\
& \left.\left.-Y_{\nu}\left(\eta_{2}^{\prime}\right) N\left(\eta_{3}^{\prime}\right)\right)-c_{33} k^{\prime}\left(J_{1}\left(\eta_{2}\right) L_{2}\left(\eta_{1}\right)-Y_{1}\left(\eta_{2}\right) L_{1}\left(\eta_{1}\right)\right)\left(M_{2}\left(\eta_{3}^{\prime}\right) N\left(\eta_{2}^{\prime}\right)-N\left(\eta_{3}^{\prime}\right) M_{2}\left(\eta_{2}^{\prime}\right)\right)\right]
\end{aligned}
$$

for the ring PT according to Fig. 1(a) and

$$
\begin{aligned}
& \Gamma_{1}=\frac{\alpha_{1}\left(\eta_{1}^{\prime}, \eta_{2}^{\prime}, \eta_{2}, \eta_{3}\right) M_{1}\left(\eta_{1}^{\prime}\right)+\alpha_{3}\left(\eta_{1}^{\prime}, \eta_{2}^{\prime}, \eta_{2}, \eta_{3}\right) M_{2}\left(\eta_{1}^{\prime}\right)}{\alpha_{2}\left(\eta_{1}^{\prime}, \eta_{2}^{\prime}, \eta_{2}, \eta_{3}\right) M_{1}\left(\eta_{1}^{\prime}\right)+\alpha_{4}\left(\eta_{1}^{\prime}, \eta_{2}^{\prime}, \eta_{2}, \eta_{3}\right) M_{2}\left(\eta_{1}^{\prime}\right)} \\
& \frac{1}{Z_{2}}=-j \omega(4 \pi b) \frac{\alpha_{2}\left(\eta_{1}^{\prime}, \eta_{2}^{\prime}, \eta_{2}, \eta_{3}\right) M_{1}\left(\eta_{1}^{\prime}\right)+\alpha_{4}\left(\eta_{1}^{\prime}, \eta_{2}^{\prime}, \eta_{2}, \eta_{3}\right) M_{2}\left(\eta_{1}^{\prime}\right)}{N\left(\eta_{1}^{\prime}\right)} \\
& \alpha_{3}\left(\eta_{1}^{\prime}, \eta_{2}^{\prime}, \eta_{2}, \eta_{3}\right)=-\alpha_{1}\left(\eta_{1}^{\prime}, \eta_{2}^{\prime}, \eta_{2}, \eta_{3}\right) \frac{\left(P_{1}\left(\eta_{1}^{\prime}\right)-P_{1}\left(\eta_{2}^{\prime}\right)\right) N\left(\eta_{1}^{\prime}\right)-\left(Q\left(\eta_{1}^{\prime}\right)-Q\left(\eta_{2}^{\prime}\right)\right) M_{1}\left(\eta_{1}^{\prime}\right)}{\left(P_{2}\left(\eta_{1}^{\prime}\right)-P_{2}\left(\eta_{2}^{\prime}\right)\right) N\left(\eta_{1}^{\prime}\right)-\left(Q\left(\eta_{1}^{\prime}\right)-Q\left(\eta_{2}^{\prime}\right)\right) M_{2}\left(\eta_{1}^{\prime}\right)} \\
& \alpha_{4}\left(\eta_{1}^{\prime}, \eta_{2}^{\prime}, \eta_{2}, \eta_{3}\right)=\frac{N\left(\eta_{1}^{\prime}\right)-\alpha_{2}\left(\eta_{1}^{\prime}, \eta_{2}^{\prime}, \eta_{2}, \eta_{3}\right)\left[\left(P_{1}\left(\eta_{1}^{\prime}\right)-P_{1}\left(\eta_{2}^{\prime}\right)\right) N\left(\eta_{1}^{\prime}\right)-\left(Q\left(\eta_{1}^{\prime}\right)-Q\left(\eta_{2}^{\prime}\right)\right) M_{1}\left(\eta_{1}^{\prime}\right)\right]}{\left(P_{2}\left(\eta_{1}^{\prime}\right)-P_{2}\left(\eta_{2}^{\prime}\right)\right) N\left(\eta_{1}^{\prime}\right)-\left(Q\left(\eta_{1}^{\prime}\right)-Q\left(\eta_{2}^{\prime}\right)\right) M_{2}\left(\eta_{1}^{\prime}\right)} \\
& \alpha_{1}\left(\eta_{1}^{\prime}, \eta_{2}^{\prime}, \eta_{2}, \eta_{3}\right)=\frac{e_{31}^{P}}{2 b} \frac{N\left(\eta_{1}^{\prime}\right)}{R\left(\eta_{1}^{\prime}, \eta_{2}^{\prime}, \eta_{2}, \eta_{3}\right)}\left[Y_{1}\left(\eta_{2}\right)\left(L_{1}\left(\eta_{2}\right)-L_{1}\left(\eta_{3}\right)\right)+J_{1}\left(\eta_{2}\right)\left(L_{2}\left(\eta_{3}\right)-L_{2}\left(\eta_{2}\right)\right)\right] \\
& \alpha_{2}\left(\eta_{1}^{\prime}, \eta_{2}^{\prime}, \eta_{2}, \eta_{3}\right)=-\frac{N\left(\eta_{1}^{\prime}\right)}{R\left(\eta_{1}^{\prime}, \eta_{2}^{\prime}, \eta_{2}, \eta_{3}\right)\left[\left(P_{2}\left(\eta_{1}^{\prime}\right)-P_{2}\left(\eta_{2}^{\prime}\right)\right) N\left(\eta_{1}^{\prime}\right)-\left(Q\left(\eta_{1}^{\prime}\right)-Q\left(\eta_{2}^{\prime}\right)\right) M_{2}\left(\eta_{1}^{\prime}\right)\right]} \\
& \times\left[c_{11}^{P} k\left(L_{1}\left(\eta_{2}\right) L_{2}\left(\eta_{3}\right)-L_{1}\left(\eta_{3}\right) L_{2}\left(\eta_{2}\right)\right)\left(Y_{\nu}\left(\eta_{2}^{\prime}\right) N\left(\eta_{1}^{\prime}\right)-K s_{-1, \nu}\left(\eta_{2}^{\prime}\right) M_{2}\left(\eta_{1}^{\prime}\right)\right)\right. \\
& \left.-c_{33} k^{\prime}\left(J_{1}\left(\eta_{2}\right) L_{2}\left(\eta_{3}\right)-Y_{1}\left(\eta_{2}\right) L_{1}\left(\eta_{3}\right)\right)\left(M_{2}\left(\eta_{2}^{\prime}\right) N\left(\eta_{1}^{\prime}\right)-N\left(\eta_{2}^{\prime}\right) M_{2}\left(\eta_{1}^{\prime}\right)\right)\right]
\end{aligned}
$$

$$
\begin{aligned}
R\left(\eta_{1}^{\prime}, \eta_{2}^{\prime}, \eta_{2}, \eta_{3}\right)= & {\left[c_{11}^{P} k\left(L_{1}\left(\eta_{2}\right) L_{2}\left(\eta_{3}\right)-L_{1}\left(\eta_{3}\right) L_{2}\left(\eta_{2}\right)\right)\left(J_{\nu}\left(\eta_{2}^{\prime}\right) N\left(\eta_{1}^{\prime}\right)-K s_{-1, \nu}\left(\eta_{2}^{\prime}\right) M_{1}\left(\eta_{1}^{\prime}\right)\right)\right.} \\
& \left.-c_{33} k^{\prime}\left(J_{1}\left(\eta_{2}\right) L_{2}\left(\eta_{3}\right)-Y_{1}\left(\eta_{2}\right) L_{1}\left(\eta_{3}\right)\right)\left(M_{1}\left(\eta_{2}^{\prime}\right) N\left(\eta_{1}^{\prime}\right)-N\left(\eta_{2}^{\prime}\right) M_{2}\left(\eta_{1}^{\prime}\right)\right)\right] \\
& +\frac{\alpha_{3}\left(\eta_{1}^{\prime}, \eta_{2}^{\prime}, \eta_{2}, \eta_{3}\right)}{\alpha_{1}\left(\eta_{1}^{\prime}, \eta_{2}^{\prime}, \eta_{2}, \eta_{3}\right)}\left[c_{11}^{P} k\left(L_{1}\left(\eta_{2}\right) L_{2}\left(\eta_{3}\right)-L_{1}\left(\eta_{3}\right) L_{2}\left(\eta_{2}\right)\right)\left(Y_{\nu}\left(\eta_{2}^{\prime}\right) N\left(\eta_{1}^{\prime}\right)-K s_{-1, \nu}\left(\eta_{2}^{\prime}\right) M_{2}\left(\eta_{1}^{\prime}\right)\right)\right. \\
& \left.-c_{33} k^{\prime}\left(J_{1}\left(\eta_{2}\right) L_{2}\left(\eta_{3}\right)-Y_{1}\left(\eta_{2}\right) L_{1}\left(\eta_{3}\right)\right) \times\left(M_{2}\left(\eta_{2}^{\prime}\right) N\left(\eta_{1}^{\prime}\right)-N\left(\eta_{2}^{\prime}\right) M_{2}\left(\eta_{1}^{\prime}\right)\right)\right]
\end{aligned}
$$


for the ring PT according to Fig. 1(b), where the functions and variables are defined by

$$
\begin{aligned}
& L_{1}(\eta)=\frac{d J_{1}(\eta)}{d \eta}+\frac{J_{1}(\eta)}{\eta} \frac{c_{12}^{P}}{c_{11}^{P}} \\
& L_{2}(\eta)=\frac{d Y_{1}(\eta)}{d \eta}+\frac{Y_{1}(\eta)}{\eta} \frac{c_{12}^{P}}{c_{11}^{P}} \\
& M_{1}(\eta)=\frac{d J_{\nu}(\eta)}{d \eta}+\frac{J_{\nu}(\eta)}{\eta} \frac{c_{13}}{c_{33}} \\
& M_{2}(\eta)=\frac{d Y_{\nu}(\eta)}{d \eta}+\frac{Y_{\nu}(\eta)}{\eta} \frac{c_{13}}{c_{33}} \\
& N(\eta)=K\left[\frac{d s_{-1, \nu}(\eta)}{d \eta}+\frac{s_{-1, \nu}(\eta)}{\eta} \frac{c_{13}}{c_{33}}\right]+\frac{e_{33}^{P}}{c_{33} \varepsilon_{33}^{P \prime}} \frac{1}{\eta} \\
& P_{1}(\eta)=\frac{e_{33}^{P}}{\varepsilon_{33}^{P \prime}} J_{\nu}(\eta)+\frac{e_{31}^{P \prime}}{\varepsilon_{33}^{P \prime}} \int_{0}^{\eta} \frac{J_{\nu}(x)}{x} d x \\
& P_{2}(\eta)=\frac{e_{33}^{P}}{\varepsilon_{33}^{P \prime}} Y_{\nu}(\eta)+\frac{e_{31}^{P \prime}}{\varepsilon_{33}^{P \prime}} \int_{0}^{\eta} \frac{Y_{\nu}(x)}{x} d x \\
& Q(\eta)=\frac{e_{33}^{P}}{\varepsilon_{33}^{P \prime}} K s_{-1, \nu}(\eta)+\frac{e_{31}^{P \prime}}{\varepsilon_{33}^{P \prime}} K \int_{0}^{\eta} \frac{s_{-1, \nu}(x)}{x} d x+\frac{1}{\varepsilon_{33}^{P \prime}} \ln \eta \\
& \eta_{1}^{\prime}=k^{\prime} r_{1}, \quad \eta_{2}^{\prime}=k^{\prime} r_{2}, \quad \eta_{3}^{\prime}=k^{\prime} r_{3} \\
& \eta_{1}=k r_{1}, \quad \eta_{2}=k r_{2}, \quad \eta_{3}=k r_{3}, \omega=2 \pi f \text {, } \\
& c_{13}=c_{13}^{P}+\frac{e_{33}^{P} e_{31}^{P \prime}}{\varepsilon_{33}^{P \prime}}, \quad c_{33}=c_{33}^{P}+\frac{\left(e_{33}^{P}\right)^{2}}{\varepsilon_{33}^{P \prime}} \text {. }
\end{aligned}
$$

The complex form of the elastic moduli $c_{11}^{P}\left(1-j / Q_{m}\right)$ and $c_{33}\left(1-j / Q_{m}\right)$ was used in the numerical calculations in order to take into account the mechanical losses. The same value of $Q_{m}$ was used for both radial vibration modes. The material parameters of PZT ceramics (type APC841) used in numerical calculations are:

$$
\begin{aligned}
& \rho=7650 \mathrm{kgm}^{-3}, \quad c_{11}^{E}=20.1 \cdot 10^{10} \mathrm{~Pa}, \\
& c_{12}^{E}=14.5 \cdot 10^{10} \mathrm{~Pa}, \quad c_{13}^{E}=10.4 \cdot 10^{10} \mathrm{~Pa}, \\
& c_{33}^{E}=13.4 \cdot 10^{10} \mathrm{~Pa}, \quad \varepsilon_{33}^{S}=770 \varepsilon_{0}, \\
& e_{31}=-5.5 \mathrm{Cm}^{-2}, \quad e_{33}=16.6 \mathrm{Cm}^{-2} .
\end{aligned}
$$

\section{Experiment}

The set of the ring PT's was made from the hard PZT, type APC $841^{11}$ of the diameters 8 and $40 \mathrm{~mm}$ and thickness $1 \mathrm{~mm}$. All samples were covered by the water soluble Ag-paste and poled homogeneously in the thickness direction at the temperature of $130^{\circ} \mathrm{C}$ and electric field of $3 \mathrm{kV} / \mathrm{mm}$ in silicone oil. Then the electrode was removed and permanent Ag-paste electrodes were deposited according to the design in Fig. 1. Radial poling was performed with the primary circuit electrodes shortened at the temperature of $130^{\circ} \mathrm{C}$ for $10 \mathrm{~min}$ in silicon oil, but with the nonhomogeneous electric field. According to the Gauss' law of electrostatics, the electric field distribution during the radial poling is inversely proportional to the distance $r$ from the ring center

$$
E(r)=\frac{U}{\ln (D / d)} \frac{1}{r}
$$

The maximum $E_{\max }$ and minimum $E_{\min }$ electric field values during the radial poling calculated according to Eq. (43) are listed in Table 1. Coercive field for APC841 was measured from hysteresis loop ${ }^{13}$ as high as $1.42 \mathrm{kV} / \mathrm{mm}$. Electric field for the radial poling step was above the coercive field for the used PZT ceramic type within the secondary transformer part only close to the inner circumference. Volume close to the outer ring's circumference was not fully poled with the field intensity below the coercive field. However, the higher voltage used for the poling resulted in the ring crack. Technologically it is very difficult to pole slender bar homogeneously as good as thin plate in thickness direction. Such nonperfect poling might influence the transformation parameters negatively.

The PTs were driven by a low-power harmonic signal (amplitude 10 V) from a function generator (HP3325A, Hewlett-Packard, Palo Alto, CA) and a resistor decade (R13000, Cosinus GmbH, Unterhaching, Germany) was connected to the secondary part of PT. At the resonance conditions and optimum load, there might be not enough power to keep constant $10 \mathrm{~V}$ amplitude at the input voltage, i.e., it is a regime of constant power for the function generator. However, the actual input and output voltages were measured simultaneously. The transformation ratio was calculated correctly as a ratio of actual voltage amplitudes. Transformation parameters were measured by the oscilloscopes (Agilent 3102A, Agilent Technologies Inc., Santa Clara, CA) for the voltage in the input and output circuit and using the current probe (CT-2, Tektronix Inc., Beaverton, OR) for the current in the input circuit.

The transformation ratio was calculated as a ratio of the input $\left(U_{1}\right)$ and output $\left(U_{2}\right)$ voltage amplitudes. The transformation efficiency was expressed as a ratio of the output and input power calculated from the measured input voltage,

Table 1. Radial poling parameters of the ring PT's (hard PZT, APC841, $d=8 \mathrm{~mm}, D=40 \mathrm{~mm}, 2 b=1 \mathrm{~mm}$ ).

\begin{tabular}{lccc}
\hline$d_{1}[\mathrm{~mm}]$ & $U[\mathrm{kV}]$ & $E_{\max }[\mathrm{kV} / \mathrm{mm}]$ & $E_{\min }[\mathrm{kV} / \mathrm{mm}]$ \\
\hline \multicolumn{4}{c}{ Inner ring electrode = INPUT } \\
20 & 12.5 & 1.80 & 0.90 \\
22 & 11.0 & 1.67 & 0.92 \\
24 & 9.3 & 1.52 & 0.91 \\
26 & 8.5 & 1.52 & 0.99 \\
\multicolumn{4}{c}{ Outer ring electrode $=$ INPUT } \\
\hline
\end{tabular}


input current and their phase shift, output voltage and output circuit resistive load $Z_{L}$.

\section{Results and Discussion}

The ring transformers of both designs [Figs. 1(a) and 1(b)] have three resonance frequencies in the radially extensional mode at about 50, 140 and $245 \mathrm{kHz}$ within the measured frequency range up to $300 \mathrm{kHz}$ - see Fig. 2 for frequency dependence of transformation ratio. The resonance frequency is not substantially dependent on the electrode diameter for the studied radial electrode design. The transformation ratios are always higher for the first and second overtone of the radially extensional vibration mode for the both no-load $\left(Z_{L}=1 \mathrm{M} \Omega\right.$, i.e., the input impedance of the multimeter or oscilloscope probe) as well as optimum load conditions see the data in Tables 2-4. The best efficiency values are met at the optimum load for the first and second overtone frequency studied. The transformation ratio shows the saturated values with increasing load. The efficiency reaches its maximum at the optimum resistive load and decreases for the smaller as well as higher loads - see Fig. 3.

The best efficiency (99\%) for the ring PT according to Fig. 1(a) is reached at the optimum load $\left(Z_{L}=10 \mathrm{k} \Omega\right)$ for the first overtone resonance on the sample with the electrode diameter of $22 \mathrm{~mm}$. It is interesting to note that such electrode

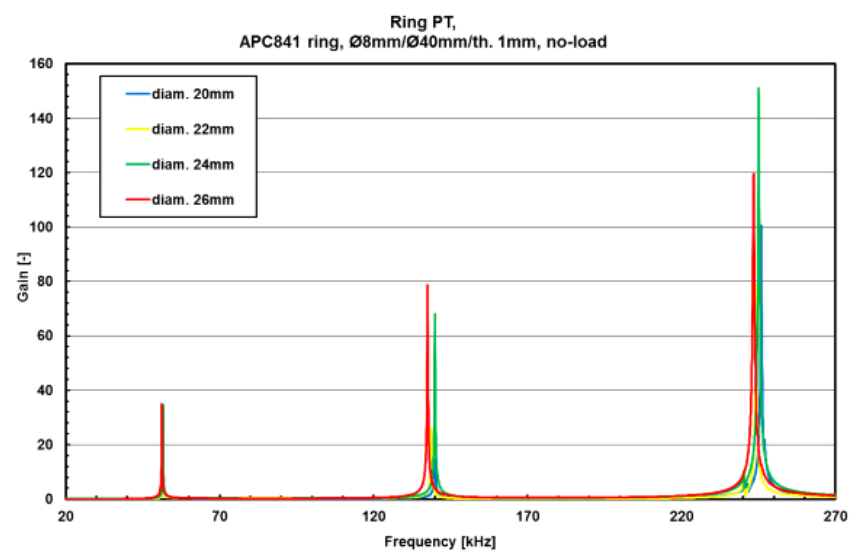

Fig. 2. Transformation ratio as a function of frequency for the ring transformer under no-load $\left(Z_{L}=1 \mathrm{M} \Omega\right)$ conditions.

Table 2. Ring PT parameters for the fundamental resonance.

\begin{tabular}{|c|c|c|c|c|c|c|}
\hline \multirow[b]{2}{*}{$d_{1}[\mathrm{~mm}]$} & \multicolumn{2}{|c|}{ No-load } & \multicolumn{4}{|c|}{ Optimum load } \\
\hline & $f_{r}[\mathrm{kHz}]$ & $U_{2} / U_{1}[-]$ & $f_{r}[\mathrm{kHz}]$ & $U_{2} / U_{1}[-]$ & $\eta[\%]$ & $Z_{L}[\mathrm{k} \Omega]$ \\
\hline 20 & 51.70 & 19.8 & 51.58 & 7.8 & 15 & 20 \\
\hline 22 & 51.40 & 17.7 & 51.28 & 5.1 & 26 & 10 \\
\hline 24 & 51.65 & 34.6 & 51.48 & 9.6 & 17 & 20 \\
\hline 26 & 51.25 & 34.9 & 51.15 & 6.8 & 19 & 10 \\
\hline \multicolumn{7}{|c|}{ INPUT $=$ outer ring } \\
\hline 24 & 52.75 & 18.6 & 52.58 & 8.4 & 6 & 20 \\
\hline
\end{tabular}

Table 3. Ring PT parameters for the first overtone resonance.

\begin{tabular}{|c|c|c|c|c|c|c|}
\hline \multirow[b]{2}{*}{$d_{1}[\mathrm{~mm}]$} & \multicolumn{2}{|c|}{ No-load } & \multicolumn{4}{|c|}{ Optimum load } \\
\hline & $f_{r}[\mathrm{kHz}]$ & $U_{2} / U_{1}[-]$ & $f_{r}[\mathrm{kHz}]$ & $U_{2} / U_{1}[-]$ & $\eta[\%]$ & $Z_{L}[\mathrm{k} \Omega]$ \\
\hline 20 & 139.95 & 14.6 & 139.49 & 2.9 & 81 & 50 \\
\hline 22 & 138.70 & 26.2 & 137.1 & 1.3 & 99 & 10 \\
\hline 24 & 139.95 & 68.0 & 138.84 & 3.5 & 96 & 10 \\
\hline 26 & 137.55 & 78.7 & 137.15 & 6.6 & 92 & 10 \\
\hline \multicolumn{7}{|c|}{ INPUT $=$ outer ring } \\
\hline 24 & 137.50 & 217.5 & 137.82 & 12.2 & 92 & 10 \\
\hline
\end{tabular}

Table 4. Ring PT parameter for the second overtone resonance.

\begin{tabular}{lccccccc}
\hline & \multicolumn{2}{c}{ No-load } & & \multicolumn{5}{c}{ Optimum load } \\
\cline { 2 - 3 } \cline { 5 - 7 }$d_{1}[\mathrm{~mm}]$ & $f_{r}[\mathrm{kHz}]$ & $U_{2} / U_{1}[-]$ & & $f_{r}[\mathrm{kHz}]$ & $U_{2} / U_{1}[-]$ & $\eta[\%]$ & $Z_{L}[\mathrm{k} \Omega]$ \\
\hline 20 & 245.90 & 100.7 & & 245.15 & 7.6 & 89 & 5 \\
22 & 244.25 & 78.7 & & 243.06 & 4.1 & 93 & 5 \\
24 & 245.10 & 151.0 & & 243.56 & 7.4 & 97 & 5 \\
26 & 243.45 & 119.4 & 242.17 & 6.1 & 97 & 5 \\
24 & 244.00 & 217.1 & 244.8 & 13.4 & 97 & 10 \\
\hline
\end{tabular}

aspect ratio corresponds to the smallest transformation ratio under optimum load condition. The experimental data for studied ring PT's $(d / D=0.2)$ shows the optimum electrode aspect ratio of $d_{1} / D \approx 0.6$ for the second overtone resonance under no-load conditions - see Fig. 4(a). The maxima of noload transformation ratios are at $d_{1} / D \approx 0.6-0.65$ for the fundamental mode and $d_{1} / D \approx 0.65$ for the first overtone as it is seen in Fig. 4(a). The optimum impedance load decreases with the resonance frequency value for the higher overtones compared to the fundamental mode as it corresponds to the capacitive nature of PT. The transformation ratio at the resonance under the optimum load increases at higher overtones.

The ring PT according to Fig. 1(b) shows higher transformation ratio under no-load conditions. Its value reaches more than 200 for the first and second overtone in the radially extensional mode. Such design also shows the higher transformation ratio at the optimum load [Fig. 3(d)] than the design according to Fig. 1(a), but the efficiency at this load is not higher. The efficiency at the optimum load is higher than $90 \%$, what is comparable to the design according to Fig. 1(a). However, the inner circumference electrode as an output [Fig. 1(b)] is not very practical for the application of such transformer, while the center hole is supposed to be a mechanical clamping point for the ring transformer.

Theoretical calculations are presented as an example how to optimize the transformation ratio as a function of the electrode dimension aspect ratio [see Fig. 4(b)] for the ring aspect ratio $d / D=0.2$. The mechanical quality factor $Q_{m}=1000$ was used in the numerical calculations. However, the mechanical quality factor measured on the 


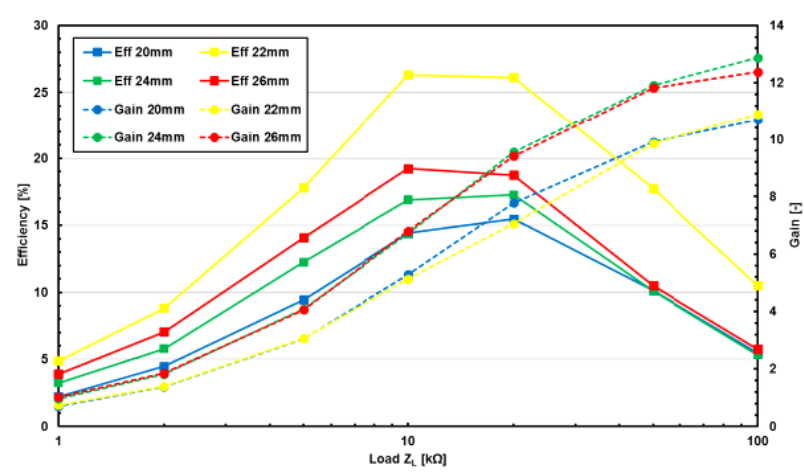

(a) Ring PT-APC 841 , ring $\varnothing 8 \mathrm{~mm} / \varnothing 40 \mathrm{~mm} / \mathrm{th} .1 \mathrm{~mm}$ INPUT=inner electrode; fundamental mode

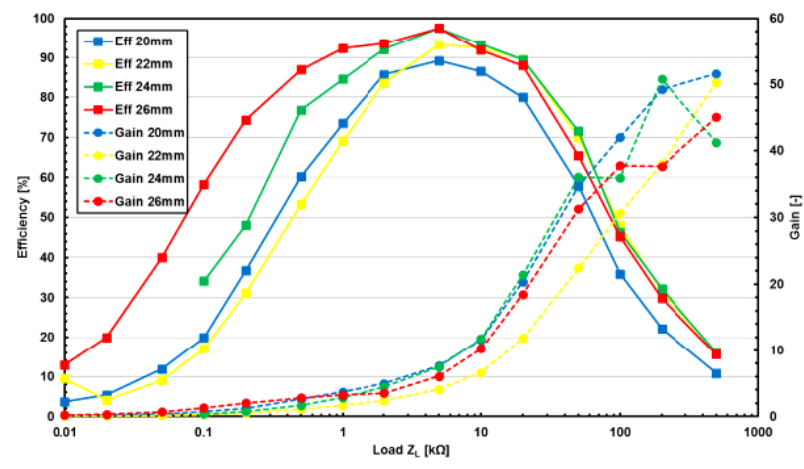

(c) Ring PT-APC841, ring Ø8mm/Ø40mm/th. $1 \mathrm{~mm}$ INPUT=inner electrode; second overtone

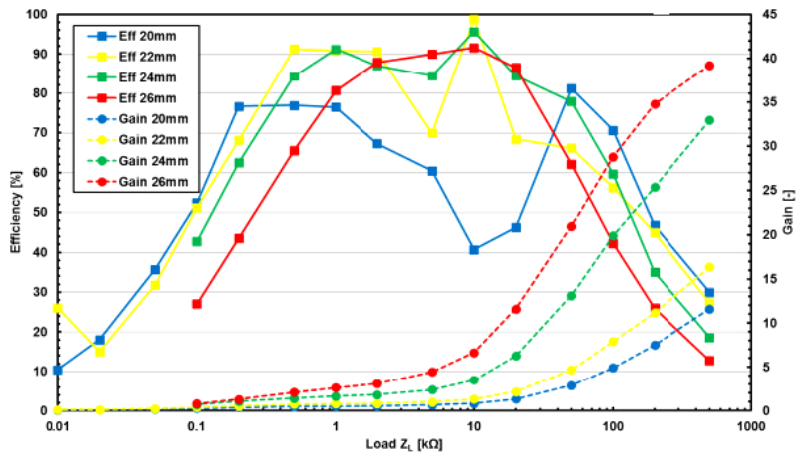

(b) Ring PT-APC841, ring $\varnothing 8 \mathrm{~mm} / \varnothing 40 \mathrm{~mm} / \mathrm{th} .1 \mathrm{~mm}$ INPUT=inner electrode; first overtone

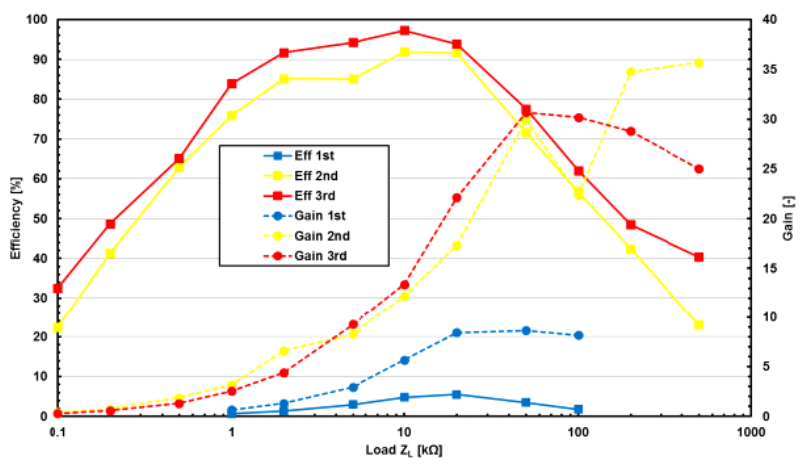

(d) Ring PT-APC841, ring $\varnothing 8 \mathrm{~mm} / \varnothing 40 \mathrm{~mm} / \mathrm{th} .1 \mathrm{~mm}$ INPUT=outer electrode $\varnothing 24 \mathrm{~mm}$

Fig. 3. Efficiency and transformation ratio as a function of resistive load $Z_{L}$ for the ring transformer - (a) fundamental mode, (b) first overtone, (c) second overtone for the electrode design according to Figs. 1(a) and 1(d) transformation parameters for the design according to Fig. 1(b).

homogeneously poled PZT resonator might be very much different from the mechanical quality factor of the nonhomogeneously poled transformer. The mechanical quality factor $Q_{m}$ is also different for the different vibration modes. ${ }^{12}$

Simulated transformation ratio values and the measured data show similar trend with respect to the resonance frequency changes with the electrode aspect ratio, fit is very good (within 5\% difference from the measured resonance frequency value). Similar results are obtained for higher overtones. Theoretical model seems to be reasonable to describe the transformer behavior, but the PT's nonhomogeneous poling and the different mechanical quality factors for the different vibration modes might introduce the differences with respect to the measured transformation ratio data.

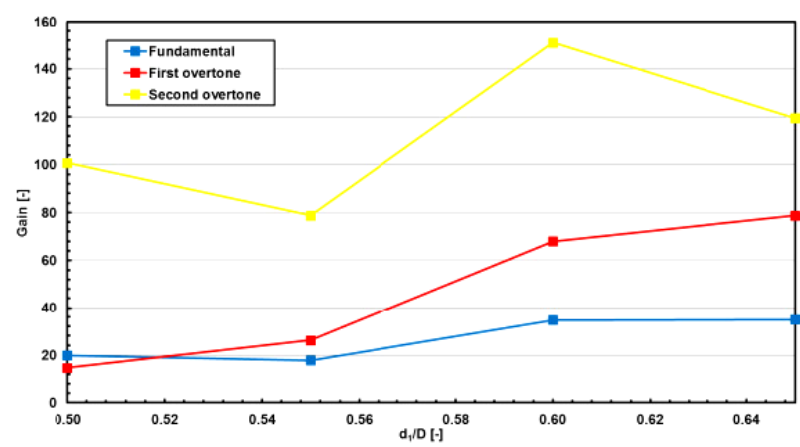

(a) Ring PT, no-load, APC841; diam. 8mm/40mm/th.1mm; INPUT=inner electrode

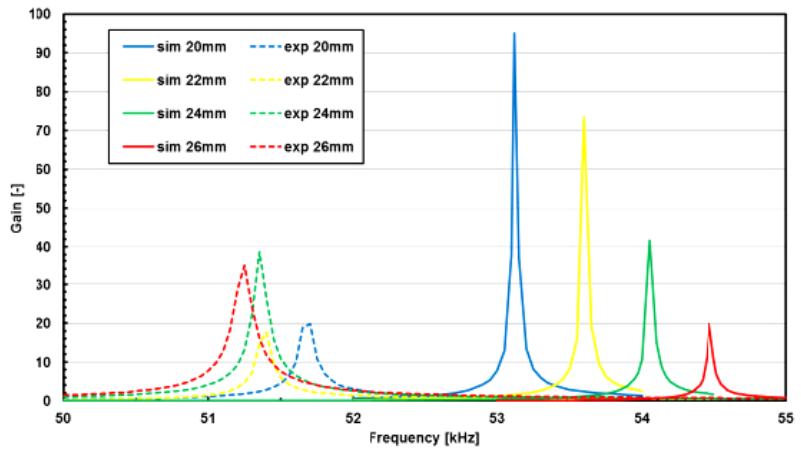

(b) Ring PT-fundamental resonance, APC841; diam. $8 \mathrm{~mm} / 40 \mathrm{~mm} / \mathrm{th} .1 \mathrm{~mm}$; INPUT=inner ring

Fig. 4. The transformation ratio as a function of the electrode dimension aspect ratio for the ring-type transformer under no-load condition (a) the first three resonances and (b) example of numerical simulations. 
Presented model is applicable for any ring transformer of studied design however the specific aspect ratios for the optimum electrode diameter might be material and ring aspect ratio (i.e., $d / D$ ) dependent. The mechanical quality coefficient $Q_{m}$ is especially an important material parameter for the vibration amplitude. Experimentally obtained resonance frequency for the peak transformation ratio is systematically smaller than its numerical simulation value as it is seen from Fig. 4(b) - span of all experimental as well as simulated resonance frequencies is less than $1.5 \mathrm{kHz}$. Similarly, the peak value of the transformation ratio differs between the experimental and numerical data. This is mainly due to the nonhomogeneous poling (and impossibility to obtain the accurate material data for the numerical simulations). Small inaccuracies of the electrode deposition by $\mathrm{Ag}$ paste are less important and do not change the vibration mode and its frequency substantially.

\section{Conclusions}

The new type of ceramic transformer was studied theoretically and experimentally for two different electrode designs. The transformation parameters were theoretically derived for the nonhomogeneously poled ring PT including mechanical losses. There are similar trends in calculated transformation ratio results like for the experimental data, but the approximation accuracy is limited to $5 \%$ by the poling nonhomogeneity and therefore unknown electromechanical parameters. The poling strategy in two subsequent steps is developed for the sample preparation. The ring transformers with the input part at inner PT's ring show the optimum electrode aspect ratio of $d_{1} / D=0.60-0.65$ in the studied samples with aspect ratio $d / D=0.2$ for the maximum noload transformation ratio of 151 . The efficiency is also much higher for the first and second overtone reaching up to $99 \%$. The ring transformer with the input part at outer PT's ring shows excellent high no-load transformation ratio of 217, the high efficiency $97 \%$ and transformation ratio 13.4 at the optimum load. However, the mechanical clamping of such a transformer at the central hole would be more difficult to prevent suppression of the vibrations.

The ring PT has very high transformation ratio allowing for its application e.g., in plasma generators under no-load transformation conditions for the plasma ignition.

\section{Acknowledgments}

This work was supported by the Czech Ministry of Education under the project LO1213.

\section{References}

${ }^{1}$ J. S. Yang, Piezoelectric transformer structural modeling - A review, IEEE Trans. Ultrason. Ferroelectr. Freq. Control 54(6), 1154-1170 (2007).

${ }^{2}$ Ch. A. Rosen, K. A. Fish and H. C. Rothenberg, Electromechanical transducer, U.S. Patent No. 2,830,274 (1958).

${ }^{3}$ P. Půlpán and J. Erhart, Transformation ratio of "ring-dot" planar piezoelectric transformer, Sens. Actuators A 140(2), 215-224 (2007).

${ }^{4}$ J. H. Hu, H. L. Li, H. L. W. Chan and C. L. Choy, A ring-shaped piezoelectric transformer operating in the third symmetric extensional vibration mode, Sens. Actuators A 88(1), 79-86 (2001).

${ }^{5}$ S.-T. Ho, Modeling and analysis on ring-type piezoelectric transformers, IEEE Trans. Ultrason. Ferroelectr. Freq. Control 54 (11), 2376-2384 (2007).

${ }^{6}$ J. S. Yang, Z. Chen and Y. Hu, Theoretical modeling of a thickness-shear mode circular cylinder piezoelectric transformer, IEEE Trans. Ultrason. Ferroelectr. Freq. Control 54(3), 621-626 (2007).

${ }^{7}$ W. Chen, Ch. Lu, J. S. Yang and J. Wang, A circular cylindrical, radially polarized ceramic shell piezoelectric transformer, IEEE Trans. Ultrason. Ferroelectr. Freq. Control 56(6), 1238-1245 (2009).

${ }^{8}$ C. F. Lü, J. S. Yang, J. Wang and W. Q. Chen, Power transmission through a hollow cylinder by acoustic waves and piezoelectric transducers with radial polarization, J. Sound Vib. 325(4-5), 989999 (2009).

${ }^{9}$ S. Lin, Z. Fu, X. Zhang, Y. Wang and J. Hu, Radially sandwiched cylindrical piezoelectric transducer, Smart Mater. Struct. 22(1), 015005 (2013).

${ }^{10} \mathrm{~S}$. Lin and S. Wang, Radially composite piezoelectric ceramic tubular transducer in radial vibration, IEEE Trans. Ultrason. Ferroelectr. Freq. Control 58(11), 2492-2498 (2011).

${ }^{11}$ APC International, Ltd., Mackeyville, USA, Available at www. americanpiezo.com.

${ }^{12} \mathrm{~K}$. Uchino, Y. Zhuang and S. O. Ural, Loss determination methodology for a piezoelectric ceramic: New phenomenological theory and experimental proposals, J. Adv. Dielectrics 1(1), 17-31 (2011).

${ }^{13}$ P. Půlpán, L. Rusin and J. Erhart, Influence of poling conditions on material properties of lead zirconate-lead titanate ceramics, Jpn. J. Appl. Phys. 47(10), 7953-7958 (2008). 